\title{
Semences et agrobiodiversité : pour une lecture ontologique des bio-communs locaux
}

Seed and agricultural biodiversity: a plea for an ontological interpretation of the local bio-commons

\section{Fabien Girard}

\section{OpenEdition}

Journals

Édition électronique

URL : http://journals.openedition.org/developpementdurable/13339

DOI : 10.4000/developpementdurable.13339

ISSN : $1772-9971$

Éditeur

Association DD\&T

Référence électronique

Fabien Girard, « Semences et agrobiodiversité : pour une lecture ontologique des bio-communs locaux », Développement durable et territoires [En ligne], Vol. 10, n¹ | Avril 2019, mis en ligne le 04 avril 2019, consulté le 01 mai 2019. URL : http://journals.openedition.org/developpementdurable/13339 ; DOI : 10.4000/developpementdurable.13339

Ce document a été généré automatiquement le 1 mai 2019.

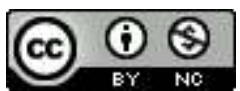

Développement Durable et Territoires est mis à disposition selon les termes de la licence Creative Commons Attribution - Pas d'Utilisation Commerciale 4.0 International. 


\section{Semences et agrobiodiversité : pour une lecture ontologique des bio- communs locaux}

Seed and agricultural biodiversity: a plea for an ontological interpretation of the local bio-commons

Fabien Girard

1 Ce travail procède de la perplexité suscitée par l'affirmation selon laquelle les semences ${ }^{1}$ auraient librement circulé pendant des siècles («libre accès »), avant l'irruption des dispositifs techniques et juridiques qui en auraient permis l'appropriation privative. La proposition se trouve d'ailleurs mobilisée par divers camps, y compris celui globalement attaché à la protection des réseaux semenciers traditionnels (e.g., Brush, 2004 ; Safrin, 2004), mais elle a aussi servi d'arme discursive dans les débats sur l'accès aux RG. Elle contredit un enseignement de la littérature anthropologique récente: "The notion of "frictionless" circulation of planting material through rural social networks and seed systems [...] is problematic » (Coomes et al., $2015: 44$ ).

2 L'ignorance (volontaire ou non) de cette proposition - qui ne veut certainement pas dire que les réseaux semenciers traditionnels sont fermés sur eux-mêmes (ibid.: 43-44) interroge ; comme si ces réseaux avaient disparu, la richesse qu'ils créent avaient été convertie en une ressource librement mobilisable, bien loin de l'image d'une série de communs locaux régis par des règles coutumières plus ou moins formalisées d'accès et d'usage. Pour quelle raison, au fond, en est-on venu à croire que les semences avaient librement circulé et que la solution aux défis actuels, notamment en termes d'érosion de l'agrobiodiversité, résiderait uniquement dans un rétablissement de cette libre circulation perdue, à travers des catégories économiques et/ou juridiques de «bien public mondial » ou « bien commun global»?

3 La thèse première de cet article est que les réseaux semenciers traditionnels, qu'on décrira bientôt comme un ensemble de bio-communs locaux, ont été, sinon détruits (remplacement massif des variétés locales par les cultivars modernes et perte corrélative 
des savoirs associés), du moins rendus invisibles dans leur dimension profonde et ainsi réduits à une simple ressource par une série de transformations paradigmatiques, ellesmêmes portées par une succession de choix politiques et juridiques.

Nous avons tous quelque chose en commun, ce qu'on appelle de manière non technique le " commun" (et nous suivrons cet usage en utilisant des guillemets) - une ressource essentielle que nous partageons et dont nous dépendons tous dans une plus ou moins grande mesure, comme nous le montre de manière criante l'érosion de la diversité des plantes cultivées. Mais cette "ressource » n'a jamais forcément le même nom (aliment, compagnon, objet rituel, RG, etc.), ni le même visage, car les rapports à la nature sont pluriels, non nécessairement instrumentaux et anthropocentrés (y compris dans les pays industrialisés : Bresnihan, 2016).

5 Il convient donc de rétablir ces multiples visages en proposant une lecture ontologique des bio-communs locaux, i.e. en redonnant une visibilité aux réseaux semenciers traditionnels et en dépassant la vision ressourciste (utilitariste) de la semence.

6 Les enjeux immédiats sont considérables. Il s'agit de préserver les réseaux semenciers traditionnels au sein desquels $70 \%$ des semences échangées circulent encore (McGuire et Sperling, 2016) et qui assurent la sécurité alimentaire d'un grand nombre de populations, notamment les plus pauvres (ibid.). Grâce au brassage de différents génotypes, ces réseaux ont assuré la distribution spatiale et sociale de la diversité génétique, morphologique et variétale, de plus en plus reconnue comme déterminante pour la productivité agricole et l'adaptation au changement climatique (Coomes et al., 2015). Enfin, les systèmes agricoles locaux et diversifiés sont mieux à même de fournir l'ensemble des micronutriments nécessaires à la santé que les chaînes d'approvisionnement mondiales (Toledo et Burlingame, 2006).

7 Un autre enjeu, tout aussi crucial, porte sur la nécessité de poser les bons diagnostics afin d'orienter de manière fine les choix politiques et législatifs. L'horizon apparemment indépassable de l'appropriation privative nous oblige, à tout le moins, à réfléchir à la manière dont pourraient être rétablis, encouragés et protégés ces bio-communs locaux à l'intérieur du cadre de contrainte actuel.

8 Avant d'aller plus loin, rappelons la propriété remarquable de la semence : elle a à la fois une dimension physique (le matériel végétal qui contient les unités fonctionnelles d'hérédité) et une dimension informationnelle (l'ADN et les valeurs socio-culturelles qui lui sont attachées) (Halewood, 2013). Dans la théorie économique, cette propriété en fait un bien faiblement rival et difficilement excluable - il s'agit donc d'un bien public potentiel (voir tableau $n^{\circ} 1$ ).

Tableau 1. Typologie économique des biens au sens de Samuelson

\begin{tabular}{|l|l|l|}
\hline & Rival & Non rival \\
\hline Excluable & Biens privés & Biens de « club » \\
\hline Non excluable & CRP (biens publics « impurs »)/commons & Biens publics (« purs ») \\
\hline
\end{tabular}

9 Par ailleurs, et surtout, les semences forment des objets bioculturels, produits d'interactions complexes entre femme/homme, communauté et écosystème, obligeant à 
prendre en compte les savoirs associés qui en ont permis l'éclosion, et les valeurs et représentations qui médiatisent les rapports entre environnement et communauté (Santilli et Emperaire, 2006 : 102). Ces caractéristiques sont particulièrement bien établies pour les RPGAA que le Traité sur les ressources phytogénétiques pour l'alimentation et l'agriculture (TIRPAA), signé en 2001, définit comme le "matériel génétique d'origine végétale ayant une valeur effective ou potentielle pour l'alimentation et l'agriculture " (TIRPAA, art. 2), et qui ont été produites par l'activité de domestication et de sélection d'innombrables générations d'agriculteurs.

10 Sur cette base, ce travail poursuit une double démonstration. La première est la suivante : en raison de l'invisibilisation des bio-communs locaux, dont il faudra montrer le processus et l'étendue, les solutions actuelles, qui entendent assurer la conservation et l'utilisation durable des RPGAA en mobilisant les catégories de «communs » ou «biens publics ", ne peuvent remplir leur objectif faute de dépasser la vision de la semence comme ressource et intégrer les différents statuts ontologiques de la semence au niveau local.

11 La seconde s'inscrit dans le prolongement de la première : quel cadre conceptuel adopter pour appréhender au mieux la complexité des bio-communs locaux, i.e. les instances où, tant dans les pays industrialisés que dans les PED, les semences paraissent « mises en commun» et "gérées» par une communauté ou un réseau de communautés d'agriculteurs?

Dans le sillage des travaux montrant les limites de la théorie du choix rationnel - même révisée (Lara, 2015) - sur laquelle est bâti le modèle ostromien, et d'études plaidant pour une meilleure prise en compte du réseau de relations sociales, émotionnelles et de pouvoirs (Nightingale, 2011:124 ; Saunders, $2014: 647$; Singleton, 2017), et des multiples connexions entre humain, non-humains et territoires (Bresnihan, 2016) intervenant dans la construction et la gestion des communs, l'article propose une relecture de la théorie des communs. Il s'efforce en particulier de rendre visible la place exacte de la semence au sein des institutions traditionnelles, les statuts ontologiques variables (attachements) qu'elle peut recevoir selon les communautés, et sa position souvent décentrée par rapport au modèle ostromien qui pense largement le cadre institutionnel de gouvernance à l'aune de l'efficience ${ }^{2}$, i.e. selon une forme unique de rationalité (Nightingale, 2011).

13 Cette double enquête s'articule autour de deux grandes séquences historiques. La première évoque la question du statut ontologique des semences, en examinant le contexte très particulier dans lequel se font jour les premières invocations du « commun » dans le domaine végétal (1). La seconde s'intéresse à la période plus récente, qui a précédé et suivi l'adoption du TIRPAA, et vient montrer, à la fois la fragilité de ce commun dit «global» et la manière dont, pour saisir les bio-communs locaux, le cadre ostromien mérite d'être repensé (2). 


\section{Premières invocations du « commun » dans un contexte d'invisibilisation des réseaux semenciers traditionnels}

\subsection{Changements de paradigmes dans la sélection des plantes : de la semence à la ressource génétique} sélection variétale à partir du XIX ${ }^{e}$ siècle. On soulignera seulement que, même si en Europe la redécouverte des lois de Mendel et les travaux de Johannsen sur les lignées pures n'ont pas immédiatement débouché sur l'artefact "gène » et la création d'un nouveau cadre expérimental destiné «à la production de masse de formes de vie stables" (Saraiva, 2010: 468-69), ils ont néanmoins servi d'arrière-plan au déploiement de nouvelles représentations du monde dont sont effacés semences, variétés locales et réseaux semenciers traditionnels, et l'appareillage de ces représentations par le système juridique lui-même.

15 Le cadre expérimental non mendélien qui se construit en Europe (particulièrement en France: Bonneuil, Thomas, 2009) permet l'émergence d'une nouvelle unité à la fois scientifique et industrielle particulière, unité dont on peut connaître et dont on prétend fixer une fois pour toutes les réactions au milieu et aux techniques culturales, dans un environnement lui-même contrôlé : la variété homogène, stable, prédictible; «variétélignée " qui se trouve placée au cœur du projet fordiste de modernisation génétique de l'agriculture. De leur côté, les variétés populations, i.e. les écotypes entretenus dans des environnements variés par les paysans depuis la naissance de l'agriculture, sont réduits à un réservoir coupé des réseaux culturels et évolutifs auxquels appartiennent les agriculteurs et leurs activités de sélection (cf. Bonneuil et Thomas, $2009: 80$ ).

Ce qui est sans doute remarquable, c'est de voir le nouveau paradigme se fixer, d'abord dans les règles d'inscription au catalogue (les trois critères de distinction, homogénéité et stabilité - DHS - et celui de la valeur agronomique et technologique - VAT - qui permet le contrôle de certaines qualités comme la force boulangère), ensuite dans celles de la propriété intellectuelle (PI). C'est précisément la Convention internationale pour la protection des obtentions végétales du 2 décembre 1961 (Conv. UPOV 1961) qui permet de cristalliser la nouvelle unité expérimentale. Ce texte vient, en effet, consacrer le paradigme « fixiste » de la variété végétale dans le système de PI qui reconnait ainsi un nouveau droit: le droit d'obtention végétale (DOV) accordé au sélectionneur sur une variété distincte, homogène et stable (Bonneuil et Thomas, 2009 : 118).

Les développements conjoints de l'ingénierie génétique et le droit de propriété permettent, à partir des années 1980, de basculer vers le paradigme du gène - unité cognitive de la sélection végétale désormais fixée. L'intégration au cadre de la PI s'accomplit d'autant plus aisément que le gène est accessible dans sa dimension moléculaire. Ramenées à des compositions chimiques, les entités biologiques peuvent alors facilement se voir étendre la jurisprudence qui a été appliquée aux composés chimiques (Calvert et Joly 2011). C'est ici que s'écrit l'histoire d'une large reconnaissance de la brevetabilité des organismes vivants (Charkrabarty 447 US 303 (1980)), puis des graines, plantes et cultures tissulaires aux États-Unis (Ex parte Hibberd 227 USPQ 
443 (1985)), et la transposition européenne du mécanisme du brevet aux séquences de gènes et aux inventions susceptibles d'être intégrées à une multitude de variétés.

\subsection{La prise de conscience de la destruction du « commun »} directement sur les pratiques et institutions. Les agriculteurs, cantonnés dans un rôle d'utilisateurs finaux dans le dispositif scientifico-industriel de progrès génétique planifié qui s'installe après-guerre, et bientôt condamnés à ne plus pouvoir échanger leurs semences et ainsi maintenir leurs activités de conservation et de renouvellement des variétés de pays, adoptent progressivement les variétés "modernes» et délaissent semences traditionnelles et savoirs associés. Cette transformation menace directement la diversité des plantes cultivées. Les sélectionneurs s'en alarment parfois précocement, qui prennent conscience d'avoir détruit « the very commons they relied on » (Saraiva, $2013: 189$ ) et de mettre ainsi en danger leur avenir. Car c'est bien le risque de compromettre le futur même de l'amélioration des plantes qui inquiète et qui motive, par exemple, l'alerte lancée dès 1936, par Harlan et Martini, dans un article resté célèbre (Harlan et Martini, 1936).

anciennes à travers un travail d'inventaire et de conservation des variétés locales qui forment de grandes collections privées et publiques (Thomas, $2006: 828$ ). Successeurs des jardins botaniques, les collections privées et publiques reconstituent un ensemble de réseaux par lesquels les obtenteurs font circuler leurs créations ainsi qu'une variabilité génétique plus large puisée dans de grands centres de diversité à l'occasion de vastes expéditions (Bonneuil et Thomas, 2009 : 217). Mais ce n'est pas assez et un projet de plus vaste ampleur doit se mettre en place.

Créée en 1945, l'Organisation des Nations unies pour l'alimentation et l'agriculture (FAO) intensifie ses efforts en faveur de la conservation des RG à partir des années 1960. Ils aboutissent à la création, en 1974, de l'Institut international des ressources phytogénétiques (IPGRI - désormais Bioversity International), rattaché au Groupe consultatif pour la recherche agricole internationale (CGIAR), la FAO n'ayant qu'un rôle de secrétariat (Andersen, 2016: 100). Les missions de prospection et de collecte s'intensifient alors de manière notable, tandis que se constituent des banques de gènes à l'échelle nationale, régionale et internationale (ibid.). Mais comme l'érosion des RPGAA atteint des niveaux sans précédent, l'IPGRI se voit reconnaître des pouvoirs considérables sur l'orientation des activités de conservation et assure, de fait, la répartition des collections entre banques (Andersen, 2016: 100). Il est alors décidé que $15 \%$ des échantillons collectés seront stockés dans les PED, tandis que $85 \%$ devront rejoindre les banques de gènes des pays développés ainsi que celles des centres internationaux de recherche agricole (IARCs) du CGIAR, pour l'essentiel situées dans les PED (ibid.).

Cette décision va contribuer à exacerber les tensions entre le Nord et le Sud, en jetant une lumière crue sur les problèmes posés par les nouveaux paradigmes de la sélection variétale et la diffusion des droits de PI sur les variétés et le matériel génétique. Contestant le rôle joué par l'IPGRI et le CGIAR, en particulier leur absence d'indépendance par rapport aux pays développés, s'inquiétant aussi d'une possible privatisation de certaines banques de gènes, certaines ONG dénoncent alors avec vigueur une double injustice qui servira à structurer le discours plus tard dominant sur la biopiraterie. 
22 La première était d'ordre économique : l'utilisation sans contrepartie des ressources phytogénétiques pour le développement de nouvelles technologies était jugée équivalente, pour le fournisseur de la ressource, au fait de «transférer la valeur d'usage du germoplasme au receveur, sans saisir l'opportunité de taxer ce que les économistes appellent la "rente de situation" qui s'attache au contrôle monopolistique sur la ressource» (Kloppenburg, 1988: 188). La seconde forme d'injustice était sociale: l'asymétrie économique était aggravée par les variétés élites développées dans les pays industrialisés et diffusées dans les PED auprès des agriculteurs à faibles revenus - circonstances de nature à ébranler les systèmes semenciers traditionnels, dont les agriculteurs dans les PED tirent indépendance et résilience (De Schutter, 2011 : 312-313).

C'est à la lueur de cette double injustice qu'il faut comprendre le large soutien apporté par les PED à une proposition de la délégation mexicaine, en 1981, de poser les bases d'une convention sur la faisabilité d'une banque de gènes internationale, qui débouchera, non sans tensions (Fowler, 1994 : 187-191), en 1983, sur une résolution importante de la FAO : l'Engagement international (EI) sur les ressources phytogénétiques (Résolution 8/83). Non contraignant et contesté, l'EI a néanmoins reçu un important soutien (113 pays signataires) (Andersen, 2016) et proposé une solution dont on a parfois salué l'audace: selon son article $1^{\mathrm{er}}$, l'instrument était «fondé sur le principe universellement accepté selon lequel les ressources phytogénétiques sont le patrimoine commun de l'humanité et devraient donc être accessibles sans restriction ».

\subsection{L'échec de la reconstruction du « commun » : le bien public mondial}

Par sa mobilisation du concept de "patrimoine de l'humanité ${ }^{3}$ ", l'EI est une contribution intéressante à la réflexion sur le statut des RPGAA. Il pointe d'emblée vers la catégorie économique du "bien public», puisque, comme on l'a dit, les RPGAA présentent potentiellement (Kaul, 2012: 40) les caractéristiques d'excluabilité difficile et de faible rivalité (voir supra). On peut même dire qu'il se réclame de la catégorie, qui se déploie au début des années 1980 en même temps que la prise de conscience de la biodiversité comme enjeu mondial (Frison, 2018b), de «bien public global» (Halewood et al., 2012: 12). Dès lors, les « bénéfices » des RPGAA sont perçus comme " universels » du point de vue des pays, de la population et des générations (actuelles et futures) (Kaul et al., 1999:2-3, 10-11) et nécessitent donc une coopération transnationale (ibid. : 14-15).

L'EI entendait mettre en place cette coopération transnationale et fournir globalement des " ressources phytogénétiques » accessibles « sans restriction », en faisant notamment lever tous les droits de PI sur les ressources. Dans sa version initiale, la catégorie « ressources phytogénétiques » incluait, en effet, non seulement les cultivars obsolètes et races de pays, mais aussi et surtout les "souches génétiques spéciales" (lignées de sélection avancées, lignées d'élite et mutants - art. 2.1). Pour atteindre ses objectifs, l'EI pouvait mobiliser deux catégories juridiques distinctes, mais qui se recoupent au moins partiellement ${ }^{4}$ : celle, évidente puisque mentionnée dans la version française de l'EI, de "patrimoine commun de l'humanité", et celle de "res communis", i.e. les "choses communes» (e.g., l'air, les eaux courantes, la mer et ses rivages) qui, par nature, n'appartiennent à personne et dont l'usage est commun à tous. Dans les deux cas, les ressources prises in globo se trouvaient placées dans une sphère d'extracommercialité, de manière à faire effectivement cesser l'emprise des droits de PI (pour les res communis : 
Chardeaux, 2006 : 78 ; pour le patrimoine commun : Birnie et al., 2009 : 197). Dans les deux cas, la qualification pouvait paraître répondre à l'une des caractéristiques des semences : leur valeur s'accroît avec l'usage - et c'est la sous-utilisation qui les menace. Elle promettait aussi de rétablir un certain équilibre nord-sud: les PED pouvaient ainsi escompter contourner les droits de PI protégeant les variétés et RG des pays développés, tout en s'attaquant également aux embargos économiques (RAFI 1988:6). Les PED pouvaient aussi espérer convaincre plus facilement les pays développés, pour certains largement tributaires des pays du Sud riches en biodiversité, et qui trouvaient eux aussi dans la qualification un moyen de contourner les embargos (RAFI, 1988 : 5). Si l'intention était louable, la qualification n'a pas permis d'atteindre les objectifs escomptés.

La catégorie de res communis - dans sa réélaboration normative (Rochfeld, 2017) - aurait dû conduire à insister sur les devoirs qui s'imposent notamment dans l'usage des choses communes (Chardeaux, 2006: 337-361 - voir aussi Brush, 2004:222), ce qui aurait dû passer, à tout le moins, par une insistance sur l'article 5 de l'EI imposant la remise d'échantillons sous réserve de "réciprocité » ou à des « conditions approuvées d'un commun accord». Toutes les potentialités de la qualification de "patrimoine commun de l'humanité » ont été réduites à néant pour cette autre raison : faute de volonté politique d'ériger, sous gestion internationale, les États en fidéicommissaires des ressources phytogénétiques (sur le trust, voir Sand, 2006) et de les charger d'assurer le respect de règles internationales (Brody, 2010 : 55-56) minimales en matière de "réciprocité ", ou encore de partage des bénéfices (Schroeder et Pogge, 2009 : 268-9; Brush, 2004 : 223) ou surtout de consentement préalable (Brush, $2004: 222-224)$, le texte a pareillement ouvert la voie à son propre travestissement.

L'échec reste fondamentalement imputable à la manière dont est pensée une nouvelle fois la semence. Alors que les débats qui précédent l'adoption de l'EI s'articulent autour d'un double enjeu de justice - accès au germoplasme, juste rétribution et rétablissement de la souveraineté des agriculteurs -, la seconde dimension est complètement absente du texte qui se concentre exclusivement sur les conditions et le régime d'accès aux ressources pour la recherche et la sélection et la compensation. Par cette méconnaissance du rôle des agriculteurs dans l'usage et l'entretien de l'agrobiodiversité, les promoteurs du texte ont contribué à maintenir l'invisibilité des réseaux semenciers traditionnels et limité la semence à une ressource appropriable.

C'est donc sans surprise que, utilisant la théorie lockéenne de la propriété fondée sur le travail des ressources naturelles (Brush, 2004 : 231) - disqualifiant ainsi toute l'innovation paysanne -, les pays industrialisés ont aisément réussi à sortir de l'article 2.1 de l'EI les variétés commerciales protégées afin de les faire échapper au principe du libre accès 5 . Ce faisant, au lieu d'entendre le «patrimoine commun » comme ce qui doit faire l'objet d'un usage et d'une jouissance dans l'intérêt de tous (y compris les générations futures), ils ont subverti la catégorie et l'ont réduite à un simple réservoir de germoplasme soumis au principe du «premier arrivé, premier servi» (Schroeder et Pogge, 2009: 268-9). Ce n'est donc qu'un demi-paradoxe que l'EI ait pu déboucher sur un renforcement sans précédent de la PI sur les semences (l'« hyperownership » décrit par Safrin : 2004), puisqu'il prépare le processus de privatisation des ressources que viennent consacrer deux textes qu'il faut lire ensemble : la Convention sur la diversité biologique (CDB), adopté au sommet de la Terre à Rio en 1992, « réaffirmant » dans son préambule (après la résolution 3/91) que « les États ont des droits souverains sur leurs ressources biologiques». Ensuite, l'accord sur les ADPIC, en 1994, et tous les accords de libre-échange subséquents qui ont accéléré le 
mouvement de diffusion des règles en matière de PI (Drahos, 2001), de mise sur le marché des variétés et de certification des semences (Wattnem, 2016).

L'accord sur les ADPIC, en particulier (not. art. 27, \$1), a conduit à une extension considérable - matérielle et géographique - du champ de la brevetabilité (en Europe, voir la directive 98/44/CE ; Girard, 2015). Les exceptions prévues au texte (27, \$3b) ont pu être utilisées par certains pays pour exclure de la brevetabilité les ressources biologiques (Van Overwalle, 2005: 589) - mais le bilatéralisme américain a parfois contrarié de telles ambitions (Drahos, 2001 ; Girard, 2018). À tout le moins, le texte, qui autorise l'exclusion des « végétaux » de la protection par brevet, a-t-il obligé les États signataires à adopter un régime juridique adéquat de protection des "variétés végétales" (la Conv. UPOV en constitue le parangon, mais il peut s'agir d'un système différent); avec cet effet direct : une large reconnaissance du DOV dans les PED depuis la fin des années 1990 (ibid.).

\section{La réflexion sur les communs du végétal : du global au local}

\subsection{La construction d'un commun " global »: le TIRPAA et sa vision ressourciste}

\subsubsection{Une première prise en compte des réseaux semenciers traditionnels}

La réflexion la plus récente sur les communs du végétal date du TIRPAA, premier instrument international entièrement consacré aux RPGAA, adopté le 3 novembre 2001 et entré en vigueur le 29 juin 2004. Sans entrer dans les détails d'un texte complexe (cf. Frison, 2018b), on en soulignera simplement ici les traits les plus saillants. L'objectif du traité est «la conservation et l'utilisation durable des [RPGAA], et le partage juste et équitable des avantages découlant de leur utilisation [...] » (art.1). Les RPGAA, qui sont une souscatégorie des RG, se voient reconnaitre un statut dérogatoire lié à leur nature spécifique (Frison, 2018b). Le traité relève lui-même plusieurs caractéristiques, dont l'importance de l'intervention humaine dans la domestication des espèces et la conservation de l'agrobiodiversité et la grande interdépendance des États en matière de RPGAA (Khoury et al., 2015).

31 En raison de leur nature spéciale, les RPGAA sont donc soumises à un régime dérogatoire au régime mis en place par la $\mathrm{CDB}$, en vertu duquel chaque État est souverain sur les ressources présentes sur son territoire et peut définir les conditions d'accès et les règles de partage des avantages qui sont ensuite fixées dans un éventuel « Accord de transfert de matériel » (ATM). Le régime dérogatoire vise à faciliter l'accès et à réduire les coûts de transaction en contournant le bilatéralisme de la CDB. Pour ce faire, le traité institue un Système multilatéral (SM) qui réalise une mise en commun virtuelle d'une liste d'espèces cultivées énumérées à l'Annexe I du traité, qui sont générées et administrées par les parties contractantes et qui relèvent du domaine public (TIRPAA, art. 11.2 - 35 espèces vivrières et 29 espèces de fourrage). Sans égard pour le nombre de ressources que chaque État rend disponibles, toute partie contractante bénéficie donc d'un accès facilité au pool de ressources au moyen d'un « Accord-type de transfert de matériel » (ATTM). L'accès ne se limite d'ailleurs pas aux seules parties contractantes, puisque ces dernières accordent également l'accès aux personnes physiques et morales relevant de la juridiction de toute partie contractante (TIRPAA, art. 12.2). Enfin, tous les chercheurs individuels et les 
institutions de recherches ont accès au RPGAA du SM, même s'ils se trouvent dans un pays qui n'a pas ratifié le traité, dès lors qu'ils signent l'ATTM.

Un aspect essentiel du TIRPAA a trait à son articulation avec le régime de la CDB, tel que complété désormais par le Protocole de Nagoya sur l'accès et le partage des avantages, adopté le 29 octobre 2010. Le TIRPAA ne s'applique qu'aux RPGAA qui sont comprises dans le SM, i.e. aux RPGAA qui figurent à l'Annexe I du traité, qui sont gérées et administrées par les parties contractantes et qui relèvent du domaine public, dès lors du moins que l'accès est justifié par l'utilisation et la conservation pour la recherche, la sélection et la formation pour l'alimentation et l'agriculture. A contrario, les RPGAA destinées à d'autres utilisations (e.g., chimiques ou pharmaceutiques) tombent sous le coup de la CDB. Surtout, lorsque les RPGAA sont "détenues " par des entreprises privées (e.g., une entreprise de sélection), des $O N G$, des communautés autochtones ou locales ou des agriculteurs, elles n'entrent pas automatiquement dans le SM et se trouvent ainsi régies par le régime de la $C D B$ et le protocole de Nagoya, et donc leurs règles en matière de consentement préalable (PIC) et de partage des avantages (voir TIRPAA, art. 12.3h; Protocole de Nagoya, art. 6.2 et 7) (Chiarolla et al., 2013 : 113-114).

\subsubsection{Une construction faussée par une logique de club}

33 Couramment décrit aujourd'hui comme un commun "global»(cf. Frison, 2018b), le TIRPAA réalise en réalité une double opération qu'il faut bien comprendre et qui conduit à réviser cette qualification : la première consiste à sortir les ressources " gérées " par les communautés locales et autochtones et les agriculteurs - qui ne sont pas dans le « domaine public » - en dehors du champ du SM. La seconde consiste à intégrer au SM une partie des RPGAA du domaine public.

Le premier aspect est un progrès notable par rapport à l'EI, puisqu'il reconnaît au moins implicitement le rôle des réseaux semenciers traditionnels et laisse un espace possible pour l'élaboration d'un régime protecteur des bio-communs locaux. Certaines stipulations visent du reste à soutenir les efforts des agriculteurs et communautés autochtones et locales dans la gestion et conservation à la ferme de leurs RPGAA. Ainsi des règles en matière de partage des avantages financiers découlant de la commercialisation d'un produit qui est une RPGAA incorporant du matériel auquel le bénéficiaire $a$ eu accès grâce au SM (TIRPAA, art. 13.2d) : les avantages doivent en premier lieu bénéficier aux agriculteurs de tous les pays, en particulier dans les PED (art. 13.3 et 18.5) (sur les faiblesses du mécanisme: Halewood et al., 2012). Ainsi encore des mécanismes de partage des avantages qui ne sont pas liés à un éventuel accès à des RPGAA, comme l'échange d'information, l'accès aux technologies et le transfert de technologies (e.g., technologies de conservation destinées aux agriculteurs des PED), de même que le renforcement des capacités (TIRPAA, art. 13a, b, c), ce qui inclut notamment le développement et le renforcement d'installations destinées à la conservation et l'utilisation durable des RPGAA. Enfin, le TIRPAA est le premier texte a reconnaître expressément «les contributions passées, présentes et futures des agriculteurs de toutes les régions du monde [...] » (Préambule) et à consacrer les «droits des agriculteurs» (art. 9.2). Le texte inclut du reste un ensemble de dispositions qui, effectivement adoptées par les États, seraient à même d'offrir aux agriculteurs et aux communautés locales et autochtones une ébauche de cadre légal protecteur (TIRPAA, art. 9.2(a) - protection des connaissances -, art. 9.2 (c) - droit de participer à la prise de décisions sur les questions relatives à la conservation et à l'utilisation durable des ressources, art. 9.3 - droit des 
agriculteurs de conserver, utiliser, échanger et vendre des semences de ferme ou du matériel de multiplication).

Mais ce premier aspect reste inséparable du second, qui en infléchit considérablement la dynamique protectrice. Le TIRPAA demeure un commun «scientifique» (Thomas, 2015 : 177) : prenant acte de l'échec du « bien public global », il cherche à rétablir un accès large aux RPGAA à l'intérieur d'un modèle de progrès agricole toujours fondé sur l'appropriation privative (inscrite au cœur du mécanisme de partage des avantages financiers) et la diffusion de variétés améliorées. L'instrument s'inscrit d'ailleurs, qu'il s'agisse de pools de brevets ou de clearing-houses, telle l'International Licensing PlatformVegetables récemment déployée par l'industrie semencière (Girard et Frison, 2018), dans un fort mouvement plus général de rétablissement de l'accès à la ressource face à la multiplication parfois problématique des droits de PI (Girard, 2015).

Il a même, dirons-nous, une logique de bien de club, puisque l'article 12.3 du traité n'accorde l'accès au pool de ressources que lorsqu'il a pour « seule fin » la conservation et l'utilisation pour la "recherche, la sélection et la formation", ce qui exclut de plano les agriculteurs et communautés locales (Frison, 2018a). Dans un tel modèle, l'accès facilité aux RPGAA incluses dans le SM constitue naturellement la première forme de partage des avantages (TIRPAA, art. 13.1), tandis que les bénéficiaires s'engagent, pour préserver cet espace, à ne revendiquer aucun droit limitant l'accès facilité aux RPGAA ou à leurs parties ou composantes génétiques, sous la forme reçue dans le cadre de l'accès facilité (TIRPAA, art. 12.3d ; ATTM, art. 6.2). Si le traité autorise la protection (par brevet ou autrement) d'un produit incorporant le matériel issu du SM, c'est à la condition qu'il ne soit plus " sous la forme reçue " (cf. Chiarolla et Jungcurt, 2011: 49) et que s'opère un partage des avantages.

Ce pool de ressources se trouve complètement coupé des réseaux socio-culturels évolutifs, comme le montrent encore les initiatives récentes et nombreuses qui visent à constituer des bases de données de ressources génétiques et génomiques et qui vont même encore plus loin en cherchant à détacher l'information des «RG », «RPGAA » ou du «matériel génétique » au sens de la CDB (art. 2) et du TIRPAA (art. 2), comme s'il s'agissait d'un donné sans attachement et sans histoire ${ }^{6}$.

Dans un tel cadre, les bio-communs locaux n'ont qu'une place marginale que reflète bien le statut des stipulations relatives aux «droits des agriculteurs »: elles relèvent de la compétence exclusive des parties contractantes (TIRPAA, art. 9.3), ont un caractère déclaratoire (Frison, 2018b) et se sont jusqu'ici heurtées à l'immobilisme des États (à quelques rares exceptions près : Girard, 2018). Comme le note aussi Halewood, la diversité gérée à la ferme est vue par le TIRPAA comme une forme de "propriété privée " (Halewood, 2013 : 297), i.e. une ressource difficilement accessible, à mille lieues de la reconnaissance des contributions passées, actuelles et futures des agriculteurs à la conservation, l'amélioration et la mise à disposition des RPGAA, et de la valeur des systèmes d'innovation collectifs portés par les agriculteurs et les communautés autochtones et locales.

\subsection{Bien appréhender les bio-communs locaux dans le domaine végétal}

Le panorama dressé jusqu'ici montre l'erreur conceptuelle (si elle n'est pas stratégie) au cœur des entreprises récentes : l'ouverture de l'accès le plus large possible à la ressource, 
qui peut sans doute se comprendre dans un contexte d'exclusivisme croissant, ne suffira pas pour atteindre les objectifs de sécurité alimentaire et d'agriculture durable. Une autre voie mérite d'être empruntée : la revisibilisation des réseaux semenciers traditionnels et la mise en évidence des ontologies particulières et situées qui lient souvent inextricablement facteurs naturels et culturels. Encore faut-il appréhender correctement ces bio-communs locaux en exposant bien le lien communauté-ressource et en réexaminant le lien communauté-institution.

\subsubsection{Mieux exposer le lien communauté-ressource}

40 Selon une définition aujourd'hui classique, le «commun" se laisse ramener à trois termes: i) une architecture de ressources; ii) une communauté qui dépend de cette ressource; iii) une organisation institutionnelle, i.e. les règles de gestion de ces ressources, en particulier les règles qui ont trait à l'appropriation (Ostrom, 1990). Dans la théorie standard, on le sait, le premier terme constitue une sorte de «substrat ", puisque, pour les biens matériels, le « bien commun » est défini par référence à une typologie qui distingue aussi : biens publics/biens de club/biens privés, en fonction des degrés de rivalité (faible-élevée) et d'excluabilité (aisée-difficile) qui caractérisent la "ressource» (voir supra, schéma $\mathrm{n}^{\circ} 1$ ). Au regard de ses caractéristiques, on l'a dit, la «RG» est, en potentialité, un bien public.

41 Il faut pourtant dépasser cette vision "essentialiste ». La nature du bien peut, en effet, déjà évoluer en fonction des dispositifs scientifico-techniques (e.g., hybrides, variétés à stérilité mâle cytoplasmique) et légaux (e.g., brevet, DOV : Girard et Noiville, 2014), ainsi que de la demande effective qui agissent sur l'excluabilité et la rivalité (Nahrath, 2015 ; voir aussi Halewood, 2013 : 295) qui font glisser le bien dans les différentes catégories du cadran.

D'autres facteurs agissent aussi sur la nature du bien, en particulier les attachements multiples entre communauté et ressource. Dans le prolongement des études menées sur les biens immatériels, il faut insister sur la manière dont le commun émerge d'une coconstruction de la communauté et de la ressource devenues inséparables (cf. Madison et al., 2010 : 672) - il n'y a pas de substrat préexistant. Il faut aussi plus largement être attentif à la façon dont le commun, comme institution, lieu et écosystème, se dégage de relations de pouvoir particulières et de rapports à l'environnement, et garde trace des attachements émotionnels particuliers à la ressource (Nightingale, 2011: 120). L'observation est essentielle pour la semence et pointe vers ce qui fait le commun pour ces biens bioculturels particulièrement complexes (Santilli et Emperaire, 2006). C'est dans un rapport de constitution mutuelle communauté-ressource, médiatisé par un tissu de normes complexes, de relations de pouvoirs, des dispositifs institutionnels préexistants, de représentations du monde, que la semence prend sa valeur et gagne son statut ontologique.

On pourrait se contenter de regarder certaines tentatives modernes de constitution de communs, telles celles portées par quelques collectifs qui composent le Réseau semences paysannes (RSP), à travers le prisme du cadre ostromien. On verrait assez bien :

i) la visée (Allaire, $2017: 1097$ );

ii) la praxis des agriculteurs-commoners qui cherchent à restaurer des races primitives ou des variétés de pays, ainsi que le savoir traditionnel nécessaire pour les sélectionner, les améliorer et les adapter aux savoirs locaux, et les conserver ; 
iii) l'institutionnalisation qui se dévoile à travers l'identification de quelques-uns des principes de conception communs aux institutions durables décrits par Ostrom (1990: 90-91), comme le montre le tableau suivant $\left(n^{\circ} 2\right)$ établi notamment à partir des travaux d'Élise Demeulenaere auprès d'un groupe constitué autour des céréales panifiables (Demeulenaere et Bonneuil, 2011, para. 1), ainsi que l'association Pétanielle et le groupe national sélection participative blé du RSP (Demeulenaere, 2018) (voir aussi : Allaire, 2017 ; Thomas et al., à paraître).

Tableau 2. Principes de conception communs aux institutions durables et bio-communs locaux

\begin{tabular}{|c|c|}
\hline $\begin{array}{l}\text { Existence de limites } \\
\text { clairement définies }\end{array}$ & $\begin{array}{l}\text { Efforts politiques et institutionnels déployés pour établir les limites } \\
\text { du groupe (règles d'accès - il peut s'agir d'une cooptation ; règles } \\
\text { d'usage - conservation, pré-multiplication et multiplication - qui } \\
\text { peuvent être différentes selon les acteurs et peuvent passer par une } \\
\text { phase « d'observation » ouvrant des droits limités) }\end{array}$ \\
\hline $\begin{array}{ll}\text { Adaptation } & \text { aux } \\
\text { conditions locales } & \end{array}$ & $\begin{array}{l}\text { Règles définissant le rapport des membres du groupe à la ressource } \\
\text { commune compte tenu des besoins (e.g., panification, polyculture } \\
\text { élevage, agriculture biologique) et des valeurs (importance du } \\
\text { terroir, rupture avec le modèle industriel, souveraineté semencière) } \\
\text { de la communauté et son terroir, en accord avec la préservation et } \\
\text { l'entretien de l'identité de la ressource (e.g., les «paysans-parrains " } \\
\text { de l'association Pétanielle s'engagent, en contrepartie des semences } \\
\text { reçues, à conserver une variété aussi pure que possible sur une } \\
\text { surface de } 3000 \mathrm{~m}^{2} \text { ) }\end{array}$ \\
\hline $\begin{array}{l}\text { Existence de dispositifs } \\
\text { de choix collectifs }\end{array}$ & $\begin{array}{l}\text { Renforcement de la «communauté de pratique" à travers la } \\
\text { discussion, l'expérimentation et parfois la coopération avec des } \\
\text { scientifiques, facteurs qui contribuent à élaborer des dispositifs de } \\
\text { choix collectifs }\end{array}$ \\
\hline $\begin{array}{l}\text { Existence de modalités } \\
\text { de surveillance du } \\
\text { comportement des } \\
\text { utilisateurs }\end{array}$ & \multirow{2}{*}{$\begin{array}{l}\text { Mécanisme de surveillance et de résolution des conflits } \\
\text { (e.g., les paysans-parrains signent une convention de parrainage et de } \\
\text { multiplication) }\end{array}$} \\
\hline $\begin{array}{l}\text { Existence } \\
\text { mécanismes de } \\
\text { résolution des conflits } \\
\text { rapides et peu coûteux }\end{array}$ & \\
\hline $\begin{array}{l}\text { Autodétermination } \\
\text { minimale reconnue par } \\
\text { les autorités extérieures }\end{array}$ & $\begin{array}{l}\text { Stratégies de plaidoyer politique pour renforcer la reconnaissance } \\
\text { étatique et internationale }\end{array}$ \\
\hline
\end{tabular}

Source : à partir de Ostrom 1990 ; Demeulenaere et Bonneuil, 2011, paragraphe. 1 ; Demeulenaere 2018

Mais on manquerait aussi un aspect important: la manière dont, sous l'institutionnalisation, dans la phase de co-construction, les subjectivités ont été transformées en même temps que le sens et la valeur de la ressource (cf. Agrawal, 2003 : 
258). Celle-ci, en particulier, devient alors, en tant que partenaire de "plein droit ", le catalyseur de l'identité de la communauté, des normes d'adhésion et de circulation au sein du collectif. Les échanges entre paysans-commoners peuvent être ainsi « socialement contraints » (Bonneuil et Demeulenaere, 2011). Dans certains collectifs, les membres se conforment ainsi aux règles qui découragent les échanges commerciaux et favorisent plutôt la logique du don/contre-don. Tout en limitant l'emprise du marché "désencastré », les valeurs sociales et culturelles qui soutiennent la communauté commandent donc elles-mêmes la nature de l'échange, à son tour largement influencée par l'identité définie de la semence. D'un côté, les règles qui président à la circulation sont étrangères à la recherche d'une gestion « efficiente » de la ressource (qui est ici la « conservation » de la ressource/son identité - Allaire, 2017 : 1097 - ou la gestion de la diversité cultivée - Thomas, 2015 : 175) : le lien entre fournisseur et utilisateur de la semence est par exemple gouverné par des normes écrites ou non écrites de confiance et de hiérarchie (e.g., les transferts ont lieu entre pairs d'égal mérite ; sans le savoir associé). D'un autre côté, et surtout, les règles sont elles-mêmes commandées par le statut ontologique de la semence : le «droit à la semence » peut être décidé en fonction de l'aptitude du candidat à être «apprivoisé » par la plante, en fonction de son aptitude à embrasser une nouvelle vision du monde où les humains et non-humains peuvent coexister (Bonneuil et Demeulenaere, 2011). Ce dernier exemple signale en creux un autre aspect difficilement visible dans la théorie des communs standard, qui est celui du rapport entre communauté et organisation institutionnelle.

\subsubsection{Réexaminer le lien communauté-institution}

Des études ethnographiques et méta-analyses aujourd'hui nombreuses (Badstue et al., 2006 ; Coomes et al., 2015 ; Jarvis et al., 2017 ; Garine et al., 2018) permettent de prolonger la réflexion précédente et d'interroger l'intentionnalité des communs et les diverses rationalités qui les traversent, en particulier dans le Sud.

Frédéric Thomas et Gilles Allaire ont tous deux étudié l'intentionnalité « conservatrice » des systèmes informels d'échange. Questionnant spécifiquement les semences gérées par les communautés du Sud, le premier considère qu'il est difficile « d'assimiler [les règles des transmissions des semences] à des communs si l'on admet que ces derniers sont des institutions sociales consciemment construites en vue de la gestion durable d'une ressource » (Thomas, 2015 : 174); tandis que le second (Allaire, 2017: 1097) estime plus largement que »[s]i la conservation d'une variété n'est pas un objectif constitué, la variété est un bien altérable, qui n'a pas une identité propre [...]» - ce qui exclut la qualification de communs au sens d'Ostrom. Frédéric Thomas conclut néanmoins que, même en l'absence de règles collectives très contraignantes régissant l'usage, subsistent ce qu'il nomme des «communs faiblement institutionnalisés » (ibid. : 175).

L'idée paraît fructueuse et nous voudrions l'approfondir en montrant que les semences gérées par les communautés locales et autochtones à travers le monde (surtout dans les $\mathrm{PED})$ répondent à ce qu'on appellera des communs à «institutionnalisation diffuse ou médiate ».

Plusieurs études convergentes informent déjà de ce que les réseaux semenciers traditionnels viennent se surajouter à des institutions existantes qui les médiatisent. Dans une étude récente, Éric Garine et al. (2018) relèvent que : " [...] farmer seed systems are an emergent property of pre-existing social forms of organisation which define the role and status of 
individuals and which pre-exist seed transactions ». La proposition se trouve confirmée par l'étude menée par Badstue et al. (2006: 268) auprès de communautés locales au Mexique. Les semences circulent toujours, en réalité, selon des règles variées qui relèvent du genre, de la parenté, de l'endogamie de la richesse, du statut social, de l'appartenance ethnique, du langage (Jarvis et al., 2016 : 192-199). Au-delà, la semence a une valeur bioculturelle qui peut aussi déterminer les canaux de circulation (e.g., féculent, accompagnement, en-cas ; chose sacrée).

En somme, ces bio-communs locaux font apparaître avec un fort effet de contraste ce que des travaux récents et provenant d'horizons académiques assez divers commencent à faire entrevoir, y compris dans des contextes occidentaux : à savoir que le commun est aussi le lieu de l'«irrationnel» (Nightingale, 2014: 120), i.e. "[...] a context wherein relationships, motivations, and emotions that have very little to do with the management of resources [are] played out » (ibid.). En d'autres termes, la rationalité formelle, celle qui se trouve tendue vers la gestion efficiente/durable de la ressource dans la théorie du choix rationnel (Singleton, 2017), si elle n'est pas absente de la structuration et la gestion d'un commun, se trouve concurrencée par d'autres formes de rationalités (voir aussi les remarques de Demeulenaere $2018: 221$ ).

On comprend ainsi mieux le reproche formulé par Gudeman au cadre ostromien: " [c]ommunities of the form I examine are not devised to serve market life; irreducibly social, they operate for themselves as they relate to self-interests and the world of trade " (Gudeman, 2001: 27). L'observation trouve un écho tout particulier dans l'étude des semences gérées par certaines communautés locales et autochtones à travers le monde. La semence y est bien plus qu'un intrant: dans le langage d'anthropologie économique de Gudeman, elle est partie intégrante de la «base » (2008: 28-31), appelée encore « commons» ou «foundation » (Gudeman, $2001: 27)$. Base et communauté s'inscrivent dans une relation dialectique où biens, services, sociabilités et identités se définissent mutuellement, où patrimoine et relations sociales lient de manière indéfectible personnes, choses et identité (Gudeman, 2008: 28). Le statut des choses qui forment la base ne peut donc jamais être complètement appréhendé en dehors de la communauté (pas de coupure nette «sujetobjet »), de ses valeurs et du type de rationalité qui lui est propre (Gudeman 2001:27; Bresnihan, $2016: 123 \mathrm{sq}$.).

51 C'est un apport significatif pour la théorie des communs, qui oblige déjà à corriger la manière de lire la gestion de la semence par les communautés locales : même si, dans beaucoup de sociétés traditionnelles, les agriculteurs expriment des besoins (e.g., conditions pédoclimatiques particulières, pratiques religieuses), ainsi que des préférences (e.g., couleur, caractéristiques post-récolte) et même s'ils font évidemment face à des événements culturaux (e.g., maladies, adaptation au changement climatique) (Jarvis et al., $2016: 4-5$ ), ils satisfont les premiers et font face aux seconds selon des règles et des principes qui sont au moins partiellement étrangers à la raison calculatrice dont la visée reste l'efficience (qu'il s'agisse du maintien de la valeur marchande ou de la conservation de la population). On pourrait certes dire que, à tout le moins, les agriculteurs cherchent à maximiser la fiabilité de l'information et de la semence en se fournissant en semence auprès de proches, de voisins, du «big man » ou encore du guérisseur. Mais même dans ce cas, une étude attentive montre que les transactions sont gouvernées par les normes qui régissent plus largement les relations sociales entre les parties engagées. En d'autres termes, et pour employer un vocabulaire sociologique, si le «capital social » paraît être mobilisé, la mobilisation du réseau de relations se lit moins comme une recherche d'efficience que 
comme un passage obligé dans un cadre de contraintes sociales, sans que l'on puisse parler de "path dependence ». C'est la base qui assure la médiation des échanges et qui, en définissant les statuts, indique en creux qui peut demander quoi et à qui. Dans un tel cadre, le choix du partenaire de l'échange doit moins à la raison instrumentale de l'agent qu'à la nécessité d'assurer la continuité de la communauté en perpétuant des normes sociales qui engagent au-delà de l'échange lui-même - et dont dépendent identité et appartenance (Coomes et al., $2015: 45,47)$. C'est ce qui fondamentalement assure la diversité et l'évolution des populations de plantes, en contribuant aux flux et migrations de gènes, en exposant les variétés à de nouveaux environnements et en contrant, au moins en partie, le développement de sous-populations propres à certains agriculteurs (Jarvis et al., 2016 : 269).

\section{Discussion et conclusion}

Figure 1. Cadre ostromien et cadre renouvelé de compréhension des communs du végétal

\section{Cadre ostromien (coordination de l'action collective)}

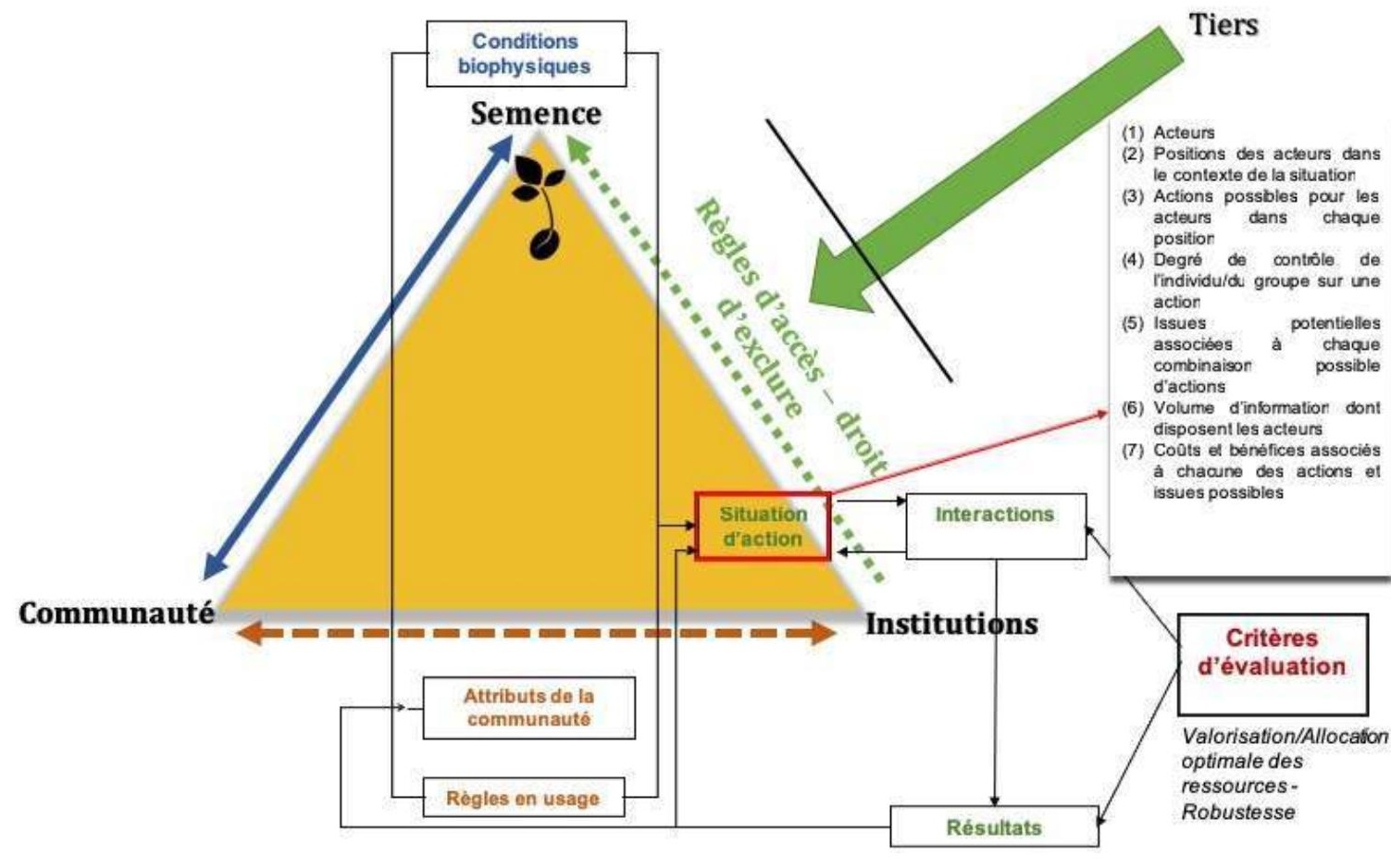




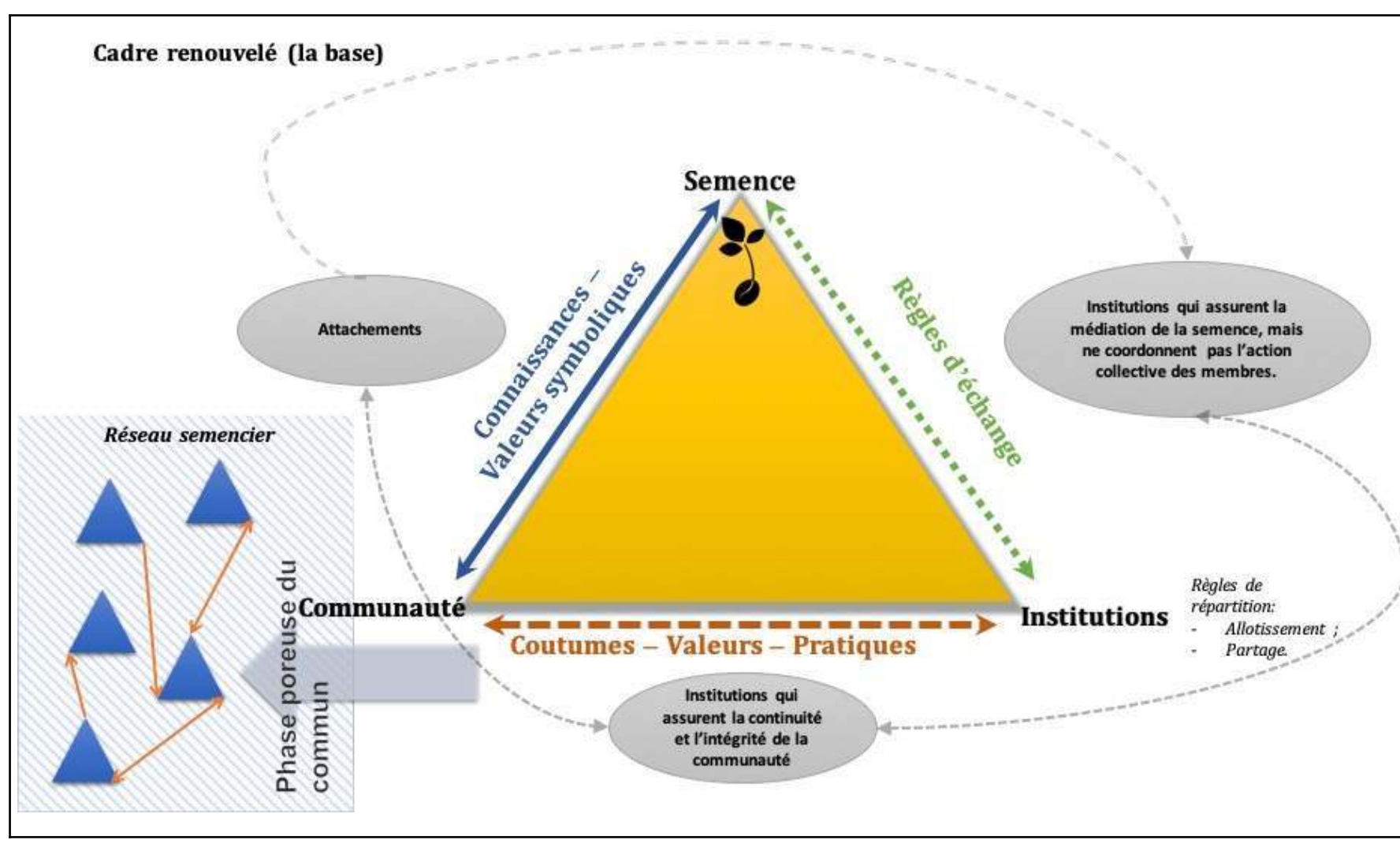

Par rapport au cadre ostromien, représenté dans sa complexité (Ostrom et Cox, 2010) en haut dans la figure 1, où l'insistance sur le rapport institutions-semences surexpose les règles destinées à régler les problèmes d'action collective («situation d'action»), un cadre renouvelé permet d'élargir la vision des bio-communs locaux aux relations communauté/institutions, puis communauté/semence. L'organisation institutionnelle est fondamentale, mais elle sert d'abord à définir la relation dialectique de la base et de la communauté dont la semence est partie intégrante (elle ne se laisse pas réduire à des «conditions biophysiques»). Les normes qui gouvernent l'échange, variables d'une communauté à l'autre, opèrent comme des cadres sociaux préexistants et non comme des arrangements institutionnels spécifiquement destinés à coordonner l'accès à la semence et sa circulation (et encore moins destinés à entretenir la diversité cultivée). La rationalité qui gouverne les échanges au sein de la base est celle de l'oikos (allotissement/partage ibid.) et reste normalement étrangère à celle qui opère sur le marché (Gudeman, 2008 : 37-38) - même si des échanges sur le marché prennent parfois place (Garine et al., 2018).

Enfin, l'élargissement de la perspective aux rapports communautés-semence permet d'apercevoir la dynamique de circulation des semences, i.e. toute l'ampleur des réseaux semenciers par lesquels les semences vont circuler et connecter les communautés. Sans doute les communautés sont-elles parfois hermétiquement fermées à l'extérieur, promptes à rejeter les free riders, les ennemis, les bioprospecteurs (le «tiers »). Pourtant, il ne faut pas outrer l'importance des règles d'accès et du droit d'exclure. Il y a toujours une face poreuse du commun où, pour reprendre l'expression de Laura Rival (2018), l'adhésion à la communauté va se négocier et où peuvent s'initier des contacts qui, sans garantir un plein accès au patrimoine bioculturel du groupe sollicité (e.g., la semence est transmise sans le savoir associé), assurent néanmoins la circulation continue et vitale du germoplasme (voir aussi Gudeman, $2008:$ 40-41). 
Compte tenu de l'importance de ces réseaux semenciers, il importe plus que jamais de maintenir les échanges entre pairs, ce qui veut dire assouplir, le cas échéant, les multiples réglementations en matière d'enregistrement des variétés et de certification des semences qui, pour certaines, incriminent les échanges semenciers entre agriculteurs (Girard, 2018). C'est l'un des aspects des droits des agriculteurs (TIRPAA, art. 9.3. ; Moore et Tymowski, 2005: 75-78) et ce que reconnait aussi, sous le nom de «droit aux semences ", la Déclaration des Nations unies sur les droits des paysans (art. 19)7.

Même si la tendance est à faire des communautés locales et autochtones et agriculteurs les intendants de la biodiversité - et de faire d'ailleurs parfois dépendre leurs droits de ce rôle, comme le postule la catégorie des droits « bioculturels " (Bavikatte, 2014) -, il serait risqué d'aller plus loin en préconisant la mise en place d'institutions destinées à mieux coordonner l'action collective dans une finalité de conservation et d'usage de la diversité cultivée. Jarvis et al. (2011) ont proposé une batterie de mesures destinées à renforcer le rôle des communautés locales et autochtones (voir tableau n ${ }^{\circ} 3$ ).

Tableau 3. Actions pouvant être entreprises pour soutenir la conservation et l'usage de variétés traditionnelles

\begin{tabular}{|c|c|}
\hline $\begin{array}{l}\text { Accroître la disponibilité } \\
\text { des semences }\end{array}$ & $\begin{array}{l}\text { - Réintroduction du matériel des collections ex situ } \\
\text { - Réintroduction de matériels présents dans des } \\
\text { environnements similaires } \\
\text { - Coopératives semencières pour la collecte, la distribution et } \\
\text { la multiplication des semences } \\
\text { - Banques de semences communautaires } \\
\text { - Banques de gènes communautaires } \\
\text { - Pépinières communautaires } \\
\text { - Trousses de diversité (diversity kit) } \\
\text { - Foires de la diversité } \\
\text { - Coupons «semences » } \\
\text { - Partage des bonnes pratiques communautaires à l'occasion } \\
\text { de visites organisées entre agriculteurs et communautés } \\
\text { - Microfinance ou microcrédit pour l'achat de semences } \\
\text { locales }\end{array}$ \\
\hline
\end{tabular}




\begin{tabular}{|c|c|}
\hline $\begin{array}{l}\text { Améliorer la qualité et la } \\
\text { disponibilité de } \\
\text { l'information }\end{array}$ & $\begin{array}{l}\text { - Création de carrés de diversité à la ferme (on-farm diversity } \\
\text { blocks) } \\
\text { - Essais en champ ou en laboratoire comparant les variétés } \\
\text { traditionnelles et modernes } \\
\text { - Registres communautaires de biodiversité } \\
\text { - Programmes d'alphabétisation pour les populations pauvres } \\
\text { et vulnérables } \\
\text { - Bases de données sur les variétés dans un format adapté aux } \\
\text { agriculteurs } \\
\text { - Mise en place de systèmes d'information et de connexions } \\
\text { Internet pour permettre l'accès des agriculteurs à } \\
\text { l'information } \\
\text { - Petites stations météorologiques connectables à Internet } \\
\text { - Programmes radiophoniques ruraux } \\
\text { - Représentations théâtrales, musicales et poétiques } \\
\text { itinérantes ayant pour thème la biodiversité } \\
\text { - Concours artistiques récompensant les groupes } \\
\text { d'agriculteurs qui dépeignent le mieux leurs idées sur la } \\
\text { conservation et l'usage des variétés traditionnelles }\end{array}$ \\
\hline $\begin{array}{l}\text { Améliorer les variétés } \\
\text { locales et leur gestion }\end{array}$ & $\begin{array}{l}\text { - Amélioration participative des plantes } \\
\text { - Utilisation de la génomique pour améliorer les populations in } \\
\text { situ } \\
\text { - Utilisation de mélanges variétaux pour réduire les parasites } \\
\text { et les maladies } \\
\text { - Amélioration des infrastructures et les méthodes de stockage } \\
\text { des semences } \\
\text { - Nettoyage/traitement des semences }\end{array}$ \\
\hline Faciliter l'accès au marché & $\begin{array}{l}\text { - Création de marchés pour les variétés traditionnelles ou les } \\
\text { produits issus de ces variétés, dont les marchés de niche } \\
\text { - Éducation et soutien financier afin d'encourager les } \\
\text { agriculteurs à développer des stratégies commerciales } \\
\text { - Institutions de microcrédit pour la mise en place de petites } \\
\text { entreprises } \\
\text { - Campagnes promotionnelles visant à attirer l'attention des } \\
\text { consommateurs et revendeurs sur l'importance de certains } \\
\text { caractères (nutrition/adaptation) } \\
\text { - Livres de cuisine et de jardinage qui promeuvent les variétés } \\
\text { traditionnelles } \\
\text { - Majorations de prix pour commerce équitable ou éco- } \\
\text { étiquetage }\end{array}$ \\
\hline
\end{tabular}

Source : à partir de Jarvis et al., 2011

Sans doute certaines de ces mesures sont-elles judicieuses et s'imposent-elles dans des situations critiques et/ou auprès de certaines communautés. Mais compte tenu des équilibres subtils qui maintiennent ces bio-communs locaux, l'opportunité de chaque décision devra être soigneusement pesée pour ne pas remettre en cause les institutions et leur fluidité qui assurent d'abord la continuité et l'identité de la communauté, et donnent son statut ontologique à la semence. 


\section{BIBLIOGRAPHIE}

Agrawal A., 2003, « Sutainable Governance of Common-Pool Resources : Context, Methods, and Politics ", Annu. Rev. Anthropol, vol. 32, p. 243-262.

Allaire G., 2017, V « Semences paysannes », in Cornu M., Orsi F., Rochfeld J. (dir.), Dictionnaire des biens communs, Paris, PUF, p. 1093-1099.

Andersen R., 2016, « Historical context : evolving international cooperation on crop genetic resources ", in Halewood M. (dir.), Farmers' Crop Varieties and Farmers' Rights. Challenges in taxonomy and law, London, New York, Routledge, p. 99-128.

Badstue L. B., Bellon M. R., Berthaud J., Juárez X., Rosas I. M., Solano A. M., Ramírez A., 2006, « Examining the Role of Collective Action in an Informal Seed System : A Case Study from the Central Valleys of Oaxaca, Mexico », Human Ecology, vol. 34, n² 2, p. 249-273.

Bavikatte S., 2014, Stewarding the earth, New Delhi, OUP.

Bresnihan P., 2016, Transforming the Fisheries : Neoliberalism, Nature, and the Commons, Lincoln, UNP.

Birnie P., Boyle A., Redgwell C., 2009, International Law and the Environment, Oxford, OUP.

Bonneuil C., Demeulenaere E., 2007, « Vers une génétique de pair à pair ? L'émergence de la sélection participative » in Charvolin F., Micoud A., Nyhart L. K. (dir.), Des sciences citoyennes ?, La Tour d'Aigues, Éd. de l'Aube, p. 122-147.

Bonneuil C., Thomas F., 2009, Gènes, pouvoirs et profits, Recherche publique et régimes de production des savoirs, de Mendel aux OGM, Versailles, Éditions Quæ.

Brody B. A., 2010, « Intellectual Property, State Sovereignty, and Biotechnology », Kennedy Institute of Ethics Journal, vol. 20, $\mathrm{n}^{\circ}$ 1, p. 51-73.

Brush S. B., 2004, Farmers Bounty : Locating Crop Diversity in the Contemporary World, New Haven, Yale University Press.

Calvert J., Joly P.-B., 2011, « How did the gene become a chemical compound : The ontology of the gene and the patenting of DNA », Social Science Information, vol. 50, $\mathrm{n}^{\circ} 2$, p. 157-77.

Chardeaux M.-A., 2006, Les choses communes, Paris, LGDJ, coll. «Bibliothèque de droit privé ».

Chiarolla C., Louafi S., Schloen M., 2013, « An Analysis of the Relationship between the Nagoya Protocol and Instruments related to Genetic Resources for Food and Agriculture and Farmers' Rights ", in Morgera E., Buck M., Tsioumani E. (dir.), The 2010 Nagoya Protocol on Access and Benefitsharing in Perspective, Leiden, Boston, Martinus Nijhoff, p. 83-122.

De Schutter O., 2011, « The Right of Everyone to Enjoy the Benefits of Scientific Progress and the Right to Food : From Conflict to Complementarity », Human Rights Quarterly, vol. 33, n² p. 304-350.

Demeulenaere E., Bonneuil C., 2011, « Des semences en partage, construction sociale et identitaire d'un collectif paysan autour de pratiques semencières alternatives », Techniques \& Cultures, vol. 57, p. 202-221. 
Demeulenaere E., 2018, « “Free our seeds !" Strategies of farmers' movements to reappropriate seeds » in Girard F., Frison C. (dir.), The Commons, Plant Breeding and Agricultural. Research Challenges for food security, agrobiodiversity and law, Oxon, Routledge, p. 210-225.

Drahos P., 2001, « BITS and BIP. Bilateralism in Intellectual Property », The Journal of World Intellectual Property, vol. 4, n 6, p. 791-808.

Fowler C., 1994, Unnatural Selection : Technology, Politics, and Plant Evolution, Yverdon, Gordon and Breach.

Frison C., 2018a, « Planting the Commons : Towards Redesigning an Equitable Global Seed Exchange » in Girard F., Frison C. (dir.), The Commons, Plant Breeding and Agricultural. Research Challenges for food security, agrobiodiversity and law, Oxon, Routledge, p. 272-289.

Frison C., 2018b, Redesigning the Global Seed Commons. Law and policy for agrobiodiversity and food security, Oxon, Routledge.

Garine E., Labeyrie V., Violon C., Wencélius J., Leclerc C., Raimond C., 2018, « Which scale to understand seed fluxes in small-scale farming societies ? Snapshots of sorghum from Africa ", in Girard F., Frison C. (dir.), The Commons, Plant Breeding and Agricultural. Research Challenges for food security, agrobiodiversity and law, Oxon, Routledge, p. 159-172.

Girard F., 2015, “"Though the Treasure of Nature's Germens Tumble All Together" : The EPO and Patents on Native Traits or the Bewitching Powers of Ideologies ", Prometheus, vol. 33, n 1, 1-23.

Girard F., 2018, " Composing the common world of the local bio-commons in the age of the Anthropocene ", in Girard F., Frison C. (dir.), The Commons, Plant Breeding and Agricultural. Research Challenges for food security, agrobiodiversity and law, Oxon, Routledge, p. 117-144.

Girard F., Frison C. (dir.), 2018, The Commons, Plant Breeding and Agricultural. Research Challenges for food security, agrobiodiversity and law, Oxon, Routledge.

Gudeman S., 2001, The anthropology of economy : community, market, and culture, Malden, Mass., Blackwell.

Gudeman S., 2008, Economy's Tension. The Dialectics of Community and Market, New York, Oxford, Berghahn Books.

Halewood M., 2013, « What kind of goods are plant genetic resources for food and agriculture? Towards the identification and development of a new global commons ", International Journal of the Commons, vol. 7, $\mathrm{n}^{\circ} 2$, p. 278-312.

Halewood M., López Noriega I., Louafi S. (dir.), 2012, Crop Genetic Resources as a Global Commons : Challenges in International Governance and Law, Oxon, Routledge.

Harlan H. V., Martini M. L., 1936, « Problems and results in barley breeding », Yearbook of Agriculture, Washington, D. C., USGPO, p. 303-346.

Jarvis D. I., Hodgkin T., Brown A. H. D., Tuxill J., López Noriega I., Smale M., Sthapit B., 2016, Crop genetic diversity in the field and on the farm : principles and applications in research practices, New Haven, Yale University Press.

Kaul I., Grunberg I., Stern M. A., 1999, Global Public goods. International Cooperation in the $21^{\text {st }}$ century, New York, PNUD, Oxford University Press.

Kaul I., 2012, « Rethinking Public Goods and Global Public Goods », in Brousseau E., Tom Dedeurwaerdere T., Siebenhüner B. (dir.), 2012, Reflexive Governance for Global Public Goods, Cambridge, MA, MIT Press, p. 37-53. 
Khoury C. K., Achicanoy H. A., Bjorkman A. D., Navarro-Racines C., Guarino L., Flores-Palacios X., Engels J. M. M., Wiersema J. H., Dempewolf H., Ramírez-Villegas J., Castañeda-Álvarez N. P., Fowler C., Jarvis A., Rieseberg L. H., Struik P. C., 2015, « Where our food crops come from : A new estimation of countries' interdependence in plant genetic resources ", CIAT Policy Brief No. 25, Cali, Centro Internacional de Agricultura Tropical (CIAT).

Kloppenburg J. K., 2004, First the seed, $2^{\mathrm{e}}$ édition, Cambridge, Cambridge University Press.

Madison M., Frischmann B., Strandburg K., 2010, « Constructing Commons in the Cultural Environment ", Cornell Law Review, vol. 95, p. 657-709.

McGuire S., Sperling L., 2016, « Seed Systems Smallholder Farmers Use », Food Security, vol. 8, n 1, p. 179-195.

Moore G., Tymowski W., (2005) Explanatory Guide to the International Treaty on Plant Genetic Resources for Food and Agriculture, Gland, Cambridge, IUCN.

Nahrath S., 2015, Vº «Bien commun », in Bourg D., Papaux A. (dir.) Dictionnaire de la pensée écologique, Paris, PUF, p. 74-78.

Nightingale A. J, 2011, « Beyond Design Principles : Subjectivity, Emotion, and the (Ir)Rational Commons », Society \& Natural Resources: An International Journal, vol. 24, n² 2, p. 119-132.

Ostrom E., 1990, Governing the Commons : The Evolution of Institutions for Collective Action, Cambridge, New York, Cambridge University Press.

Ostrom E., Cox M., 2010, « Moving beyond panaceas : a multi-tiered diagnostic approach for social-ecological analysis », Environmental Conservation, vol. 37, nº 4, p. 451-463.

RAFI, 1988, A Report on Germplasm Embargoes, RAFI Communique, Pittsboro, Rural Advancement Fund International.

Rival L., 2018, « An Anthropological Lens on Property and Access : Gudeman's Dialectics of Community and Market ", in Girard F., Frison C. (dir.), The Commons, Plant Breeding and Agricultural. Research Challenges for food security, agrobiodiversity and law, Oxon, Routledge, p. $147-158$.

Rochfeld, J., 2017, « V Chose commune (approche juridique) », in Cornu, M., Orsi, F., Rochfeld, J. (dir.), Dictionnaire des biens communs, Paris, PUF, p. 176-185.

Safrin S., 2004, « Hyperownership in a Time of Biotechnological Promise : The International Conflict to Control the Building Blocks of Life ", American Journal of International Law, vol. $98, \mathrm{n}^{\circ} 4$, p. 641-685.

Santilli J., Emperaire L., 2006, « A agrobiodiversidade e os direitos dos agricultores indígenas e tradicionais », in Ricardo B., Ricardo F. (dir.), Povos Indígenas no Brasil : 2001-2005, São Paulo, Instituto Socioambiental, p. 101-103.

Saraiva T., 2013, « Breeding Europe : Crop Diversity, Gene Banks, and Commoners » in Disco N., Kranakis E. (dir.), Cosmopolitan Commons. Sharing Resources and Risks across Borders, Cambridge, MA, MIT Press, p. 187-211.

Saunders F. P., 2014, « The promise of common pool resource theory and the reality of commons projects ", International Journal of the Commons, vol. 8, $\mathrm{n}^{\circ}$ 2, p. 636-656.

Schroeder D., Pogge T., 2009, « Justice and the convention on biological diversity », Ethics \& International Affairs, vol. 23, $\mathrm{n}^{\circ}$ 3, p. 265-280.

Singleton B. E., 2017, « What's missing from Ostrom ? Combining design principles with the theory of sociocultural viability », Environmental Politics, vol. 26, n 6, p. 994-1014. 
Thomas F., 2006, « Biodiversité, biotechnologies et savoirs traditionnels. Du patrimoine commun de l'humanité aux ABS (access to genetic resources and benefit-sharing) », Revue Tiers Monde, vol. $4, \mathrm{n}^{\circ} 188$, p. 825-842.

Thomas F., 2015, « Droits de propriété industrielle et "communs" agricoles. Comment repenser l'articulation entre domaine public, biens collectifs et biens privés ", in Vanuxem S. et Guibert Lafaye C. (dir.), Repenser la propriété, un essai de politique écologique, Aix-en-Provence, PUAM.

Thomas F., Labatut J., Allaire G. (à paraître) « Variétés végétales et races animales. De l'accès libre à l'appropriation, et à la (re)mobilisation des communs ».

Toledo, A., Burlingame B., 2006, « Biodiversity and Nutrition : A Common Path toward Global Food Security and Sustainable Development ", Journal of Food Composition and Analysis, vol. 19, n -6-7, p. 477-483.

Van Overwalle G., 2015, « Protecting and Sharing Biodiversity and Traditional Knowledge : Holder and User Tools », Ecological Economics, vol. 53, p. 585-607.

Wattnem T., 2016, «Seed Laws, Certification and Standardization : Outlawing Informal Seed Systems in the Global South », The Journal of Peasant Studies, vol. 43, $\mathrm{n}^{\circ}$ 4, p. 850-867.

\section{NOTES}

1. Le terme de semence(s) vise ici : les « semence(s)» lato sensu, i.e. les semences véritables, ainsi que les plantules et les propagules; les ressources phytogénétiques pour l'alimentation et l'agriculture (RPGAA) ; les ressources génétiques (RG); le matériel génétique ou le germoplasme.

2. L'Institutional Analysis and Development Framework développé par Ostrom offre un cadre d'analyse très riche. L'agent type y est doté d'une rationalité « limitée », puisqu'il agit dans un contexte d'information incomplète et qu'il reste faillible. En particulier, la partialité de l'information tient au fait que, à rebours des postulats la théorie néoclassique, l'agent, chez Ostrom, n'est pas nécessairement un maximisateur, et peut donc poursuivre d'autres buts (altruistes, coopératifs). La morale de l'agent n'est pas toujours conséquentialiste (utilitarisme hédoniste), puisque Ostrom admet un déontologisme. Ostrom a du reste proposé une lecture fine des espaces d'interaction sociale où les individus échangent des biens et services et délibèrent, les fameuses «arènes d'action " au sein desquelles se donne à voir une "situation d'action », i.e. là où se décident les actions (sous les variables d'informations, de préférences et de stratégies) qui produisent conjointement des résultats. S'ajoutent aussi des variables exogènes - attributs du monde biophysique, normes et valeurs partagées par la communauté et règles en usage - qui montrent bien que l'agent n'est pas un atome, mais un individu pris dans des relations sociales et contraint par des déterminants institutionnels multiniveau (Ostrom et Cox, 2010). Néanmoins, le critère d'évaluation des formes d'organisation locale, par exemple des systèmes socio-écologiques (SES), reste l'efficience, i.e. la valorisation et l'allocation optimale des ressources, ce qui inclut dans le cas précis la robustesse (dynamique d'adaptation) et une certaine forme de redistribution, mais promeut une forme unique de rationalité qu'on qualifiera de formelle (« moyen-fin »), car tournée vers les objectifs économiques et écologiques. Rationalité qui procède de l'idée qu'il faut «shape individual economic behavior by delimiting and constructing spheres of activity that are measurable and thus manageable » (Bresnihan, 2016: 128).

3. Il y avait une divergence entre le texte français de la résolution, qui visait le «patrimoine commun de l'humanité » et le texte anglais qui ne mentionnait que le "heritage of mankind». C'est la résolution 4/89 qui introduit la notion de « common heritage of mankind » dans le texte anglais. 
4. Sur les liens, parfois contestés, entre patrimoine commun et res communis, voir Chardeaux, $2006: 225-228$.

5. Résolution $4 / 89$.

6. Voir en particulier DivSeek, Global Open Data for Agriculture and Nutrition, the Research Data Alliance et le Breeding API. La grande question en cours est celle de l'applicabilité des règles d'accès et de partage des avantages de la CBD et du TIRPAA (Frison, 2018b).

7. UN, General Assembly, United Nations Declaration on the Rights of Peasants and Other People Working in Rural Areas, Human Rights Council, Thirty-ninth session, 10-28 September 2018, A/ HRC/39/L.16.

\section{RÉSUMÉS}

Les réseaux semenciers traditionnels - les bio-communs locaux - ont été, sinon détruits, du moins rendus invisibles dans leur dimension profonde, et ainsi réduits à une simple ressource par une série de transformations paradigmatiques. Les solutions passées ou actuelles, qui mobilisent les catégories de bien public global ou de commun, ne parviennent pas à assurer la conservation et l'utilisation durable des RPGAA faute de dépasser la vision de la semence comme ressource et intégrer les différents statuts ontologiques de la semence au niveau local. Le texte plaide pour un dépassement de cette vision et pour une reconceptualisation du cadre des communs du végétal. Positionné au niveau local, l'effort de renouvellement conceptuel devrait insister sur les liens entre communautés et ressources, la valeur ontologique de la semence, et mieux souligner le rôle des institutions traditionnelles dans la circulation des semences.

Farmers' seed networks - the local bio-commons - have been, if not dismantled, at least made invisible in their inner dimension and downgraded to a resource status following a series of paradigmatic shifts. Past or current solutions, which rest on the categories of global public good or on the commons, fail to ensure the conservation and sustainable use of PGRFA. Notably, they have been unable to go beyond the conception of seed as a resource and to account for the various ontological status conferred upon it at the local level.

While advocating a broader approach, the text calls for a re-conceptualization of the plant commons framework. Relying on a local-level approach, the new conceptual framework should emphasize the link between communities and resources, the ontological value of seed, and better underline the role of traditional institutions in the circulation of seed.

\section{INDEX}

Keywords : farmers' seed networks, commons, genetic resources, communities, ontologies

Mots-clés : réseaux semenciers traditionnels, communs ; ressources génétique, communautés, ontologies 


\section{AUTEUR}

\section{FABIEN GIRARD}

Fabien Girard est maître de conférences en droit privé (UGA, CRJ) et Associate Research Fellow à la Maison Française d'Oxford. Ses travaux se situent à l'intersection de la propriété, l'innovation végétale et l'agrobiodiversité.

fabien.girard@univ-grenoble-alpes.fr 